\title{
Dark $Z$ implication for flavor physics
}

\section{Fanrong $\mathbf{X u}$}

Institute of Physics, Academia Sinica, Taipei, Taiwan 11529, R.O. China

Department of Physics, Jinan University, Guangzhou 510632, P.R. China

Kavli Institute for Theoretical Physics China, Chinese Academy of Sciences, Beijing 100190, P.R. China

E-mail: frxu@hep1.phys.ntu.edu.tw

ABSTRACT: Dark $Z$ /dark photon $\left(Z^{\prime}\right)$ is one candidate of dark force carrier, which helps to interpret the properties of dark matter (DM). Other than conventional studies of DM including direct detection, indirect detection and collider simulation, in this work we take flavor physics as a complementary approach to investigate the features of dark matter. We give an exact calculation of the new type of penguin diagram induced by $Z^{\prime}$ which further modifies the well-known $X, Y, Z$ functions in penguin-box expansion. The measurement of rare decays $B \rightarrow K^{(*)} \mu^{+} \mu^{-}$and $B_{s} \rightarrow \mu^{+} \mu^{-}$at LHC, together with direct CP violation $\varepsilon^{\prime} / \varepsilon$ in $K \rightarrow \pi \pi$ as well as $K_{L} \rightarrow \mu^{+} \mu^{-}$, are used to determine the parameter space. The size of coupling constant, however, is found to be $\mathcal{O}(1)$ which is much weaker than the known constraints.

Keywords: Rare Decays, B-Physics, Kaon Physics

ARXIV EPRINT: 1504.07415 


\section{Contents}

1 Introduction 1

2 The model 2

3 The $Z^{\prime}$ effect in FCNC processes 4

$3.1 Z^{\prime}$ penguin 4

3.2 The modification to $X, Y, Z$ function 5

4 Phenomenology $\quad 7$

$\begin{array}{lll}4.1 & B_{q} \rightarrow \mu^{+} \mu^{-} & 7\end{array}$

$4.2 B \rightarrow K^{(*)} \mu^{+} \mu^{-} \quad 8$

$4.3 K_{L} \rightarrow \mu \bar{\mu} \quad 9$

$4.4 \frac{\varepsilon^{\prime}}{\varepsilon} \rightarrow \mu \mu \bar{l}$

5 Results and discussion $\quad 10$

A The calculation of $Z^{\prime}$ penguin $\quad 12$

A.1 The $\epsilon$ component 12

$\begin{array}{lll}\text { A.2 The } \epsilon_{Z} \text { component } & 14\end{array}$

\section{Introduction}

Dark matter (DM) constitutes about $27 \%$ of the energy-matter budget of universe, significantly more than $5 \%$ of baryonic matter [1]. Nevertheless, the exact nature of DM is kept mysterious so far. Yet it is still unclear whether DM can be described by scalar, fermion, vector or even graviton.

Among various DM candidates, dark photon has been the one of particular interest. The idea was initialed in 1980s [2], and developed in recent years [3-15]. Suppose there is an extra $\mathrm{U}(1)_{D}$ group besides $\mathrm{SM}$ gauge group, under which all the SM interactions are invariant. The gauge boson of $\mathrm{U}(1)_{D}$, named dark photon, interacts with $\mathrm{SM} \mathrm{U}(1)_{Y}$ gauge boson via a kinetic mixing term. It helps to explain the astrophysical observation of positron excesses [16], as well as other astrophysical phenomenology such as supernova bounds [17] and Big Bang Nucleosynthesis [18]. The direct search of dark photon, for example XENON 100, has put a very strong constraint [19]. For $e^{+} e^{-}$collider, a recent search performed at BaBar shows a null result, neither finds nor rules out dark photon [20]. A further experiment, the Heavy Photon Search (HPS) experiment located at Jefferson Lab [21], is designed to search dark photon in the mass range $20 \mathrm{MeV}$ to $1 \mathrm{GeV}$ as well as the related coupling. However, it has been realized recently that a simple dark photon is 
not favored by $3.6 \sigma$ deviation of muon anomalous magnetic moment [22]. Given the fact that dark photon model is the extreme case when the parameter to describe $Z-Z^{\prime}$ mass mixing is closed in a more generic dark $Z$ model, it is necessary to extend dark photon to dark $Z$ which is the working frame of this paper.

Flavor physics is not only taken as a platform for precise test of SM, but also plays an important role in indirect search of new physics (NP) beyond Standard Model (SM). Great progresses have already been made since LHC runs. For example, it was hoped for decades that NP might exist in zero crossing point $q_{0}^{2}$ of the differential branching ratio of $B \rightarrow K^{*} \mu^{+} \mu^{-}$(see for example [23]) but finally turned out tiny NP effect [24]. Taken DM theory as one kind of ordinary NP theory, flavor physics then would provide as a complementary way for conventional approaches of DM study, including direct detection, indirect detection and collider production. Based on the great achievement made in Run I, the LHC has already started its Run II in 2015. Then it would be interesting, and timely, to connect together these two different fields, dark matter and flavor physics. A similar effort can also be found in [25].

The paper is organized as follows. In section 2 , we will briefly set up the dark $Z$ model. An exact result of $Z^{\prime}$ penguin and further modifications to $X, Y, Z$ functions are given in section 3. Some typical processes which are affected by the $Z^{\prime}$ including the recently measured $B \rightarrow K^{*} \mu^{+} \mu^{-}$and $B_{s} \rightarrow \mu^{+} \mu^{-}$, as well as $\varepsilon^{\prime} / \varepsilon$ and $K_{L} \rightarrow \mu^{+} \mu^{-}$are discussed in section 4 , also the relevant formulas are given therein. In section 5 the obtained numerical results are shown, based on which we will make a discussion. The details of $Z^{\prime}$ penguin calculation can be found in appendix.

\section{The model}

Suppose there exits an extra U(1) group, other than SM U(1), what it brings in phenomenology is an interesting question. It was considered how electromagnetic charge is shifted by this extra U(1) group in the initial paper [2]. Until recently it becomes popular to take this $\mathrm{U}(1)$ gauge boson as a DM candidate.

Under the dark group, notated as $\mathrm{U}(1)_{D}$, all the SM interactions are invariant. The connection between dark photon with SM particles is from a kinetic mixing term, leading to the effective Lagrangian [15]

$$
\mathcal{L}=-\frac{1}{4} \hat{B}_{\mu \nu} \hat{B}^{\mu \nu}+\frac{1}{2} \frac{\epsilon}{\cos \theta_{W}} \hat{B}_{\mu \nu} \hat{Z}^{\prime \mu \nu}-\frac{1}{4} \hat{Z}_{\mu \nu}^{\prime} \hat{Z}^{\prime \mu \nu},
$$

where $\theta_{W}$ is Weinberg angle, $\hat{Z}^{\prime}$ and $\hat{B}$ are dark photon and SM $B$ field with the corresponding field strength

$$
\hat{B}_{\mu \nu}=\partial_{\mu} \hat{B}_{\nu}-\partial_{\nu} \hat{B}_{\mu}, \quad \hat{Z}_{\mu \nu}^{\prime}=\partial_{\mu} \hat{Z}_{\nu}^{\prime}-\partial_{\nu} \hat{Z}_{\mu}^{\prime},
$$

and the mixing of gauge bosons is mimicked by parameter $\epsilon$, which is supposed to be small and need to be determined. The convention above, in gauge interaction state, is not 
diagonalized. By redefining fields as

$$
\left(\begin{array}{c}
Z_{0}^{\prime} \\
B
\end{array}\right)=\left(\begin{array}{cc}
\sqrt{1-\frac{\epsilon^{2}}{c_{W}^{2}}} & 0 \\
-\frac{\epsilon}{c_{W}} & 1
\end{array}\right)\left(\begin{array}{c}
\hat{Z}^{\prime} \\
\hat{B}
\end{array}\right),
$$

the Lagrangian is then rotated to a diagonal form

$$
\mathcal{L}=-\frac{1}{4} B_{\mu \nu} B^{\mu \nu}-\frac{1}{4} Z_{0, \mu \nu}^{\prime} Z_{0}^{\prime \mu \nu}
$$

Note here the field after rotation with a subscript 0 differs from the one before rotation with a hat. In SM the $B$ field can be projected to photon and $Z$ after spontaneous symmetry breaking (SSB). Incorporating $Z_{0}^{\prime}$, the related neutral gauge fields are shifted,

$$
\begin{aligned}
A & =\hat{A}-\epsilon \hat{Z}_{0}^{\prime} \\
Z_{0} & =\hat{Z}_{0}+\epsilon \tan \theta_{W} \hat{Z}_{0} \\
Z_{0}^{\prime} & =\hat{Z}_{0}^{\prime} .
\end{aligned}
$$

The rotation does not change the definition of $Z_{0}^{\prime}$, however, photon and $Z$ field are modified indeed. Due to this modification of gauge fields, the interaction between $Z_{0}^{\prime}$ and ordinary matter is induced, which is named as dark photon model.

When $Z_{0}^{\prime}-Z_{0}$ mass mixing is considered, the simple dark photon model is then extended to dark $Z$ model. Generally speaking, the mass of $Z^{\prime}$ could either be added by hand which is called Stückelberg mechanism [26] (the origin of Stückelberg photon, for example, is discussed in string theory [27]) or by applying Higgs mechanism, see [15] as an example. In this paper, we shall adopt the treatment of $Z^{\prime}$ mass in the latter case, without involving the details of the mechanism itself. After the neutral gauge bosons obtain mass after SSB, a further rotation is required after the one in eq. (2.3) for diagonalising mass matrix

$$
\left(\begin{array}{l}
Z \\
Z^{\prime}
\end{array}\right)=\left(\begin{array}{cc}
\cos \zeta & -\sin \zeta \\
\sin \zeta & \cos \zeta
\end{array}\right)\left(\begin{array}{l}
Z_{0} \\
Z_{0}^{\prime}
\end{array}\right)
$$

where the rotation angle $\zeta$ is model dependent and analytically might be complicated, but numerically should be small, (for example, see [15]). Now combine together the two rotations eq. (2.3) and eq. (2.6), the modifications to photon and $Z$ by dark $Z$ shows

$$
\begin{aligned}
& A_{\mu}=\hat{A}_{\mu}-\epsilon Z_{\mu}^{\prime} \\
& Z_{\mu}=\cos \zeta \hat{Z}_{0, \mu}-\epsilon_{Z} Z_{\mu}^{\prime} \approx \hat{Z}_{0, \mu}-\epsilon_{Z} Z_{\mu}^{\prime} .
\end{aligned}
$$

Formally the shift of neutral fields in dark photon model is characterised by two independent parameters $\epsilon$ and $\epsilon_{Z}$, respectively. In fact $\epsilon_{Z}$ also has a lengthy analytical expression based on detailed model. The equivalent $\epsilon_{Z}$ defined to replace the complicated structure brings the convenience. The dark $Z$ field here and also hereafter is denoted as $Z^{\prime}$, with original SM field denoted with a hat. The two coupling constants together with $Z^{\prime}$ mass constitute the unique 3 model parameters of dark $Z$, which could be measured in experiments. 
Apparently the interactions between $Z^{\prime}$ and SM particles are simply induced by the shifted neutral gauge field. Explicitly, we show how $Z^{\prime}$ couples to SM fermions,

$$
\mathcal{L}_{Z^{\prime} f f}=-\left(\epsilon e J_{\mathrm{em}}^{\mu}+\epsilon Z \frac{g}{2 \cos \theta_{W}} J_{\mathrm{NC}}^{\mu}\right) Z_{\mu}^{\prime}
$$

in which the SM electrical current and weak neutral current are

$$
\begin{aligned}
J_{\mathrm{em}}^{\mu} & =Q_{f} \bar{f} \gamma^{\mu} f, \\
J_{\mathrm{NC}}^{\mu} & =\left(T_{3 f}-2 Q_{f} \sin ^{2} \theta_{W}\right) \bar{f} \gamma^{\mu} f-T_{3 f} \bar{f} \gamma^{\mu} \gamma_{5} f,
\end{aligned}
$$

where $f$ stands for fermions with corresponding electric charge $Q_{f}$, isospin $T_{3 f}= \pm \frac{1}{2}$. With both vector coupling and axial-vector coupling, $Z^{\prime}$ behaves as a light version of $Z$ and heavier version of photon. For the coupling of $Z^{\prime}$ and other gauge bosons, it has both " $Z$ component" and " $A$ component" sized by $\epsilon$ and $\epsilon_{Z}$ respectively.

\section{The $Z^{\prime}$ effect in FCNC processes}

As current energy frontier, the LHC brings plentiful opportunities for flavour physics which dominated by flavour changing neutral current (FCNC) processes. In this section, we will investigate these processes of meson physics in dark $Z$ model. It is known in SM FCNC processes are induced at loop level. Conventionally the Feynman diagrams contributing to FCNC can be classified to three point penguin diagram and four point box diagram. If NP exists, the new interaction brought in by NP will modify parts/all of these SM penguin and box contribution. Within the $Z^{\prime}$ model working frame, however, this modification is only applied in photon penguin and $Z$ penguin, keeping box diagram contribution unchanged. To make the new effect more distinguishable, we extract these modifications alone and name it as $Z^{\prime}$-penguin specifically.

\section{$3.1 \quad Z^{\prime}$ penguin}

We take $b \rightarrow s Z^{\prime}$ as an example, noting similar result can be applied to $b \rightarrow d Z^{\prime}$ and $s \rightarrow d Z^{\prime}$ when necessary conditions are satisfied.

In Feynman-t' Hooft gauge there are totally 10 Feynman diagrams giving contributions to $b \rightarrow s Z^{\prime}$. During our realistic calculation, we group two of the external leg corrections and replace them by an effective vertex shown in figure 1 , with $Z^{\prime}$ inserted in either two legs. The four self-energy diagrams, effectively two, are then shown as $(g)$ in figure 2, together with the remaining six ordinary three point diagrams given as $(a)$ to $(f)$.

As mentioned above the full result can be decomposed into " $A$ component" (or $\epsilon$ component) and " $Z$ component" (or $\epsilon_{Z}$ component). The $\epsilon$ component is same as $b \rightarrow s \gamma$ while the latter one is similar to $b \rightarrow s Z$. For photon penguin, there are two types of effective verteices corresponding to real and virtual photon. In below since we focus on semileptonic processes thus only the virtual photon vertex is taken into account. In order to keep the final result the same structure as photon penguin, the $\epsilon_{Z}$ component differs 


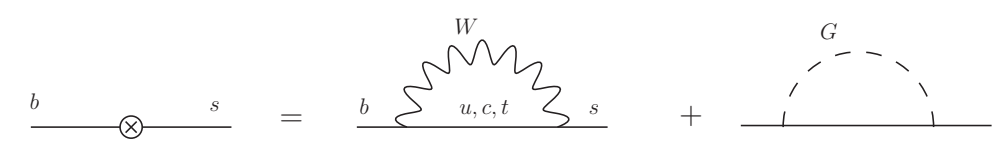

Figure 1. The effective vertex for $b \rightarrow s$ transition.

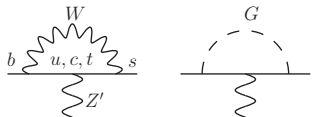

(a)

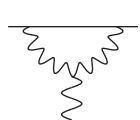

(c)

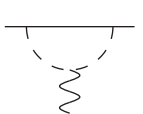

$(d)$

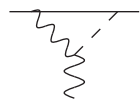

$(e)$

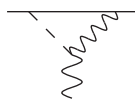

$(f)$

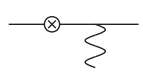

$\sum \otimes-$

Figure 2. The Feynman diagrams contributing to $b \rightarrow s Z^{\prime}$ in Feynman-t' Hooft gauge. Figure $(a)$ to $(f)$ are three point diagrams while figure $(g)$ is from the correction to external leg where the effective vertex denoted by a cross is explained in figure 1 .

from SM $Z$ penguin by neglecting dipole term contribution. An exact calculation, shown in appendix $\mathrm{A}$, gives the $Z^{\prime}$ penguin vertex as

$$
\left.\bar{s} \Gamma^{\mu} b\right|_{Z^{\prime}}=i \lambda_{i} \frac{G_{F}}{\sqrt{2}} \frac{e}{8 \pi^{2}} H_{0}\left(x_{i}\right) \bar{s}\left(q^{2} \gamma^{\mu}-q^{\mu} \phi\right)\left(1-\gamma_{5}\right) b,
$$

in which $\lambda_{i}=V_{i b} V_{i s}^{*}(i=u, c, t), x_{i}=\frac{m_{i}^{2}}{m_{W}^{2}}$ and $q$ is outgoing momentum carried by gauge boson. The vertex function $H_{0}\left(x_{i}\right)$ consisting of photon component function $D_{0}\left(x_{i}\right)$ and newly calculated $Z$ component function $\tilde{D}_{0}\left(x_{i}\right)$, are characterised by $\epsilon$ and $\epsilon_{Z}$, giving

$$
\begin{aligned}
H_{0}(x)= & \epsilon D_{0}(x)+\epsilon_{Z} \tilde{D}_{0}(x) \\
D_{0}(x)= & -\frac{4}{9} \ln x+\frac{-19 x^{3}+25 x^{2}}{36(x-1)^{3}}+\frac{x^{2}\left(5 x^{2}-2 x-6\right)}{18(x-1)^{4}} \ln x \\
\tilde{D}_{0}(x)= & -\frac{1}{s_{W} c_{W}}\left[\frac{34 x^{3}-141 x^{2}+147 x-58}{216(-1)^{3}}+\frac{\left(-3 x^{4}+18 x^{3}-27 x^{2}+19 x-4\right) \ln x}{36(x-1)^{4}}\right. \\
& \left.+c_{W}^{2}\left(\frac{-47 x^{3}+237 x^{2}-312 x+104}{108(x-1)^{3}}+\frac{\left(3 x^{4}-30 x^{3}+54 x^{2}-32 x+8\right) \ln x}{18(x-1)^{4}}\right)\right],
\end{aligned}
$$

with $s_{W}=\sin \theta_{W}, c_{W}=\cos \theta_{W}$. Note during evaluating, the light down type quark is supposed to be massless and the momentum transfer is small, comparable to light quark mass. We also assume the light $Z^{\prime}$ mass smaller than the mass threshold for muon pair production, which guarantees no $Z^{\prime}$ resonance is produced when final state of charged lepton is muon. Also in this work we will not touch electron and neutrino as the lepton final state for the production of $Z^{\prime}$ when $m_{Z^{\prime}}$ is above electron threshold. Nevertheless, it is safe to neglect $m_{Z^{\prime}}$ in this paper.

\subsection{The modification to $X, Y, Z$ function}

In the state-of-the-art effective Hamiltonian approach, physical observables can be factorized into short distance (SD) and long distance (LD) contribution. The SD part is 
treated in perturbative theory while LD hadronic matrix resorts to various methods including lattice QCD. The SD contribution gives various combination of penguin diagrams and box diagrams and leads to he so-called $X, Y, Z$ functions, which also depends on both the theoretical working frame and the calculated physical observable. For example, $Y$ is to characterize the Wilson coefficient in the effective Hamiltonian of $B_{q} \rightarrow \ell^{+} \ell^{-}$, and for a more complicated process $B \rightarrow K^{*} \mu^{+} \mu^{-}$more functions are involved. In the frame of $Z^{\prime}$ model, we have already discussed a new $Z^{\prime}$ penguin in previous sector. Before combining detailed phenomenology, to include $Z^{\prime}$ contribution in standard $X, Y, Z$ functions systematically is now our target,

In SM the $X, Y, Z$ functions are obtained via combing $b s \gamma, b s Z$, box diagram vertex (see [28]), and now we need to contain $b s Z^{\prime}$ vertex. The amplitudes of $b \rightarrow s \bar{\ell} \ell$ (or $\bar{s} b \rightarrow \bar{\ell} \ell$ ) mediated by different gauge bosons are in the form of

$$
\begin{aligned}
i \mathcal{M}_{\gamma} & =i \lambda_{i} \frac{G_{F}}{\sqrt{2}} \frac{\alpha}{2 \pi} D_{0}\left(x_{i}\right)(\bar{s} b)_{V-A}(\bar{\ell} \ell)_{V} \\
i \mathcal{M}_{Z} & =i \lambda_{i} \frac{G_{F}}{\sqrt{2}} \frac{\alpha}{2 \pi s_{W}^{2}} 2 C_{0}\left(x_{i}\right)\left[v_{f}(\bar{s} b)_{V-A}(\bar{L} L)_{V}-a_{f}(\bar{s} b)_{V-A}(\bar{L} L)_{A}\right] \\
i \mathcal{M}_{Z^{\prime}} & =i \lambda_{i} \frac{G_{F}}{\sqrt{2}} \frac{\alpha}{2 \pi s_{W}^{2}} 2 C_{0}\left(x_{i}\right)\left[\frac{s_{W} H_{0}\left(x_{i}\right)}{4 c_{W} C_{0}\left(x_{i}\right)} v_{f}^{\prime}(\bar{s} b)_{V-A}(\bar{L} L)_{V}-\frac{s_{W} H_{0}\left(x_{i}\right)}{4 c_{W} C_{0}\left(x_{i}\right)} a_{f}^{\prime}(\bar{s} b)_{V-A}(\bar{L} L)_{A}\right]
\end{aligned}
$$

with $L=(\ell, \nu)$ and the coupling of $Z f f$ and $Z^{\prime} f f$

$$
\begin{array}{ll}
v_{f}=T_{3 f}-2 Q_{f} s_{W}^{2}, & a_{f}=T_{3 f}, \\
v_{f}^{\prime}=\epsilon \cdot 2 Q_{f} s_{W} c_{W}+\epsilon_{Z}\left(T_{3 f}-2 Q_{f} s_{W}^{2}\right), & a_{f}^{\prime}=\epsilon_{Z} T_{3 f} .
\end{array}
$$

For convenience $Z$ and $Z^{\prime}$ contribution can be put together in a compact form by showing exact final state

$$
\begin{aligned}
i \mathcal{M}_{Z Z^{\prime}}^{\bar{\ell} \ell} & =i \lambda_{i} \frac{G_{F}}{\sqrt{2}} \frac{\alpha}{2 \pi s_{W}^{2}} C_{0}\left(x_{i}\right)\left[\left(-1+4 s_{W}^{2}\right)\left(1+\delta_{1}\right)(\bar{s} b)_{V-A}(\bar{\ell} \ell)_{V}+\left(1+\delta_{2}\right)(\bar{s} b)_{V-A}(\bar{\ell} \ell)_{A}\right] \\
i \mathcal{M}_{Z Z^{\prime}}^{\bar{\nu} \nu} & =i \lambda_{i} \frac{G_{F}}{\sqrt{2}} \frac{\alpha}{2 \pi s_{W}^{2}} C_{0}\left(x_{i}\right)\left[\left(1+\delta_{2}\right)(\bar{s} b)_{V-A}(\bar{\nu} \nu)_{V}-\left(1+\delta_{2}\right)(\bar{s} b)_{V-A}(\bar{\nu} \nu)_{A}\right]
\end{aligned}
$$

in which we have introduced two parameters

$$
\delta_{1}=\frac{s_{W} H_{0}\left(x_{i}\right)}{4 c_{W} C_{0}\left(x_{i}\right)}\left[\epsilon \frac{4 c_{W} s_{W}}{1-4 s_{W}^{2}}+\epsilon_{Z}\right], \quad \delta_{2}=\epsilon_{Z} \frac{s_{W} H_{0}\left(x_{i}\right)}{4 c_{W} C_{0}\left(x_{i}\right)} .
$$

Incoperating box diagram contribution, the Wilson coefficients of operators $Q_{9}^{\overline{\ell \ell}}=(\bar{s} b)_{V-A}$ $(\bar{\ell} \ell)_{V}, Q_{10}^{\bar{\ell} \ell}=(\bar{s} b)_{V-A}(\bar{\ell} \ell)_{A}$ as well as quark-neutrino operator $Q_{V-A}^{\bar{\nu} \nu}=(\bar{s} b)_{V-A}(\bar{\nu} \nu)_{V-A}$ can be explicitly extracted as

$$
\begin{aligned}
C_{9}^{\bar{\ell} \ell} & =\lambda_{i} \frac{G_{F}}{\sqrt{2}} \frac{\alpha}{2 \pi s_{W}^{2}}\left[s_{W}^{2} \cdot 4\left(Z_{0}\left(x_{i}\right)+\Delta Z_{0}\left(x_{i}\right)\right)-\left(Y_{0}\left(x_{i}\right)+\Delta Y_{V}\left(x_{i}\right)\right)\right] \\
C_{10}^{\bar{\ell} \ell} & =\lambda_{i} \frac{G_{F}}{\sqrt{2}} \frac{\alpha}{2 \pi s_{W}^{2}}\left[Y_{0}\left(x_{i}\right)+\Delta Y_{A}\left(x_{i}\right)\right] \\
C^{\bar{\nu} \nu} & =\lambda_{i} \frac{G_{F}}{\sqrt{2}} \frac{\alpha}{2 \pi s_{W}^{2}}\left[X_{0}\left(x_{i}\right)+\Delta X_{0}\left(x_{i}\right)\right]
\end{aligned}
$$


in which the $X, Y, Z$ functions are given in the combination of penguin and box diagrams $X_{0}(x)=C_{0}(x)-4 B_{0}(x), Y_{0}(x)=C_{0}(x)-B_{0}(x), Z_{0}(x)=C_{0}(x)+\frac{1}{4} D_{0}(x)$. The corresponding corrections to $X, Y, Z$ are then given as

$$
\begin{array}{ll}
\Delta Y_{V}(x)=\delta_{1} C_{0}(x), & \Delta Z_{0}(x)=\delta_{1} C_{0}(x), \\
\Delta X_{0}(x)=\delta_{2} C_{0}(x), & \Delta Y_{A}(x)=\delta_{2} C_{0}(x) .
\end{array}
$$

Especially, we find that the modification to $Y$ has two types due to different Dirac structure of lepton pair in the operators, while modification to $X$ and $Z$ function are in a fixed way. The modifications can be written in a more explicit form as

$$
\begin{aligned}
\Delta X_{0}(x) & =\frac{1}{4} t_{W} \epsilon_{Z} H_{0}(x) \\
\Delta Y_{A}(x) & =\frac{1}{4} t_{W} \epsilon_{Z} H_{0}(x) \\
\Delta Y_{V}(x) & =\left[\epsilon \frac{s_{W}^{2}}{1-4 s_{W}^{2}}+\epsilon_{Z} \frac{t_{W}}{4}\right] H_{0}(x) \\
\Delta Z_{0}(x) & =\left[\epsilon \frac{s_{W}^{2}}{1-4 s_{W}^{2}}+\epsilon_{Z} \frac{t_{W}}{4}\right] H_{0}(x) .
\end{aligned}
$$

In the limit of $\epsilon \rightarrow 0, \delta_{1}=\delta_{2}$, the modifications are identical. However, if $\epsilon_{Z} \rightarrow 0$ (exactly dark photon model case), leading to $\Delta X_{0}=\Delta Y_{A}=0$, then the phenomenology is much more tedious.

\section{Phenomenology}

The physical observables can be classified into two types in dark $Z$ model. One type relating to box diagram, like the mass difference of neutral meson, is not modified by $Z^{\prime}$. The other one involving photon and $Z$ penguins, such as the direct $\mathrm{CP}$ violation in $K \rightarrow \pi \pi$, does change. In this section, we will choose several typical processes to see a generic effect of $Z^{\prime}$ on flavour physics.

\section{$4.1 \quad B_{q} \rightarrow \mu^{+} \mu^{-}$}

It has been hoped for decades that NP might be unfolded in rare decay $B_{s} \rightarrow \mu^{+} \mu^{-}$. However, no hint of NP appeared in $B_{s} \rightarrow \mu^{+} \mu^{-}$mode from LHC Run I data, given by the full combination results of CMS and $\mathrm{LHCb}[29]$

$$
\begin{aligned}
& \mathcal{B}\left(B_{s} \rightarrow \mu^{+} \mu^{-}\right)=\left(2.8_{-0.6}^{+0.7}\right) \times 10^{-9} \\
& \mathcal{B}\left(B_{d} \rightarrow \mu^{+} \mu^{-}\right)=\left(3.9_{-1.4}^{+1.6}\right) \times 10^{-10} .
\end{aligned}
$$

Though $B_{s} \rightarrow \mu^{+} \mu^{-}$turns out to be SM-like, there remains a hope for NP in the much rarer mode $B_{d} \rightarrow \mu^{+} \mu^{-}$(for example, see [30]).

Due to the precise measurement of decay $B_{s} \rightarrow \ell^{+} \ell^{-}$is now realistic, one should consider the effect of sizeable width difference $\Delta \Gamma_{s}$ in $B_{s}^{0}-\bar{B}_{s}^{0}$ oscillation. The theoretical 
formula has to be corrected to compare with measured branching ratio [31, 32] which is denoted with a bar,

$$
\mathcal{B}\left(B_{s}^{0} \rightarrow \ell^{+} \ell^{-}\right)=\left[\frac{1-y_{s}^{2}}{1+\mathcal{A}_{\Delta \Gamma}^{\ell^{+} \ell^{-}} y_{s}}\right] \overline{\mathcal{B}}\left(B_{s}^{0} \rightarrow \ell^{+} \ell^{-}\right)
$$

where $y_{s} \equiv \frac{\Delta \Gamma_{s}}{2 \Gamma_{s}} \equiv \frac{\Gamma_{L}^{(L)}-\Gamma_{L}^{(s)}}{2 \Gamma_{s}}, \mathcal{A}_{\Delta \Gamma}^{\ell^{+} \ell^{-}}=\frac{R_{H}^{\ell^{+} \ell^{-}}-R_{L}^{\ell^{+} \ell^{-}}}{R_{H}^{\ell+\ell^{-}}+R_{L}^{\ell^{+} \ell^{-}}}$. It is known $\mathcal{A}_{\Delta \Gamma}^{\ell^{+} \ell^{-}}=1$ in SM [32], thus

$$
\mathcal{B}\left(B_{s}^{0} \rightarrow \ell^{+} \ell^{-}\right)=\left(1-y_{s}^{2}\right) \overline{\mathcal{B}}\left(B_{s}^{0} \rightarrow \ell^{+} \ell^{-}\right) .
$$

The latest estimation of parameter $y_{s}$ is $y_{s}=0.069 \pm 0.006$ given in [35]. Note in the dark photon model, the relation of eq. (4.3) does not change. While for the rarer $B_{d} \rightarrow \ell^{+} \ell^{-}$ decay, the effect from oscillation in $B^{0}-\bar{B}^{0}$ can be neglected thus we do not take this correction.

The (uncorrected) SM branching ratio of $B_{q} \rightarrow \ell^{+} \ell^{-}$is induced by $Z$ penguin and hence depends on $Q_{10}$, (see ref. [36]). Now incorporating $Z^{\prime}$-penguin, which gives a similar component as $Z$, leads to

$$
\mathcal{B}\left(B_{q} \rightarrow \ell^{+} \ell^{-}\right)=\tau\left(B_{q}\right) \frac{G_{F}^{2}}{\pi}\left(\frac{\alpha}{4 \pi s_{W}^{2}}\right)^{2} f_{B}^{2} m_{\ell}^{2} m_{B} \sqrt{1-\frac{4 m_{\ell}^{2}}{m_{B}^{2}}} \eta_{\text {eff }}^{2}\left|\lambda_{t}\left(Y_{0}\left(x_{t}\right)+\Delta Y_{A}\left(x_{t}\right)\right)\right|^{2}
$$

with $\eta_{\text {eff }}=0.9882 \pm 0.0024$ which takes into account NNLO QCD correction and NLO electroweak correction [36]. Apparently the reason why only $\Delta Y_{A}$ contributes, is exact with the same reason why photon penguin contribution vanishes.

\section{$4.2 B \rightarrow K^{(*)} \mu^{+} \mu^{-}$}

The quest for NP in $B \rightarrow K^{(*)} \mu^{+} \mu^{-}$has been performed for a long time. In the beginning the zero crossing-point $q_{0}^{2}$ is of the first priority, however, $q_{0}^{2}$ turns out to be compatible with SM prediction finally from the released LHC data. The remaining possibility for NP in this mode, the $P_{5}^{\prime}$ problem, requires more data to confirm. Meanwhile for the $B \rightarrow K \ell^{+} \ell^{-}$ channel, there is a NP hint, so called $R_{K}$ problem, which violates lepton universality.

The theoretical study for this channel has been developed for around 30 years, including multi-loop calculation of Wilson coefficients at high energy. The most matured theoretical treatment in low energy to the semileptonic decays are based on QCDF. It is not necessary to repeat the whole long story in this work. Instead, we would like to simply focus on $C_{9}$ and $C_{10}$ to see how the data constrain the NP parameter space. In dark $Z$ model, the modification of $C_{9}$ and $C_{10}$ due to the dark $Z$ contribution is

$$
\begin{aligned}
\Delta C_{9}\left(x_{i}\right) & =4 \Delta Z_{0}\left(x_{i}\right)-\frac{\Delta Y_{V}\left(x_{i}\right)}{s_{W}^{2}} \\
\Delta C_{10}\left(x_{i}\right) & =\frac{\Delta Y_{A}\left(x_{i}\right)}{s_{W}^{2}}
\end{aligned}
$$

Driven by new data many efforts have been made to extract the information hidden inside the two coefficients. In [37] a model-independent fit was taken based on $B \rightarrow X_{s} \ell^{+} \ell^{-}$, 
$B \rightarrow X_{s} \gamma, B \rightarrow K^{*} \gamma$ and $B \rightarrow K^{*} \mu^{+} \mu^{-}$, we will adopt their constraints on $\Delta C_{9}$ and $\Delta C_{10}$ at $2 \sigma^{1}$

$$
\begin{aligned}
-1.5<\operatorname{Re}\left(\Delta C_{9}\right) & <1.2, & -2.8<\operatorname{Im}\left(\Delta C_{9}\right) & <2.8 \\
-1<\operatorname{Re}\left(\Delta C_{10}\right) & <1.5, & -3<\operatorname{Im}\left(\Delta C_{10}\right) & <3 .
\end{aligned}
$$

In our scenario, the NP only exists in the change of real part of $C_{9 / 10}$.

\section{$4.3 \quad K_{L} \rightarrow \mu \bar{\mu}$}

The branching ratio of $K_{L} \rightarrow \mu \bar{\mu}$ contains LD and SD contribution. The calculation of LD contribution remains a challenge in theory. Combining a latest theoretical LD estimation and experimental bound [39], ${ }^{2}$ the constraint to $\mathrm{SD}$ is

$$
\mathcal{B}\left(K_{L} \rightarrow \mu^{+} \mu^{-}\right)_{\mathrm{SD}} \leq 2.5 \times 10^{-9} .
$$

The branching ratio from SD (see ref. [40])

$$
\mathcal{B}\left(K_{L} \rightarrow \mu^{+} \mu^{-}\right)_{\mathrm{SD}}=\kappa_{\mu}\left[\frac{\operatorname{Re}\left(\lambda_{c}\right)}{\left|V_{u s}\right|} P_{c}\left(Y_{K}\right)+\frac{\operatorname{Re}\left(\lambda_{t}\right)}{\left|V_{u s}\right|^{5}} \eta_{Y} Y_{0}\left(x_{t}\right)\right]^{2}
$$

with $\lambda_{q}=V_{q s}^{*} V_{q d}(q=c, t)$ and $\kappa_{\mu}=(2.009 \pm 0.017) \times 10^{-9}\left(\frac{\left|V_{u s}\right|}{0.225}\right)^{8}, P_{c}\left(Y_{K}\right)=(0.115 \pm$ $0.018)\left(\frac{0.225}{\left|V_{u s}\right|}\right)^{8}$ [41], QCD correction factor $\eta_{Y}=1.012$ [42], now is modified as

$$
\mathcal{B}\left(K_{L} \rightarrow \mu^{+} \mu^{-}\right)_{\mathrm{SD}}=\kappa_{\mu}\left[\frac{\operatorname{Re}\left(\lambda_{c}\right)}{\left|V_{u s}\right|} P_{c}\left(Y_{K}\right)+\frac{\operatorname{Re}\left(\lambda_{t}\right)}{\left|V_{u s}\right|^{5}} \eta_{Y}\left(Y_{0}\left(x_{t}\right)+\Delta Y_{A}\left(x_{t}\right)\right)\right]^{2}
$$

by including the dark $Z$ contribution.

\section{$4.4 \quad \frac{\varepsilon^{\prime}}{\varepsilon}$}

Historically two approaches, operator production expansion (OPE) method and penguinbox expansion (PBE) method, are adopted for the study of direct CP violation in $K \rightarrow \pi \pi$, which involves all the QCD penguin and electroweak penguin in SM. For the phenomenology study here, we make use of the simple analytical formula based on the PBE method [43]. By modifying relevant parts due to the dark $Z$ effect, an updated formula ${ }^{3}$ to depicted $\varepsilon^{\prime} / \varepsilon$ is

$$
\operatorname{Re} \frac{\varepsilon^{\prime}}{\varepsilon}=a \operatorname{Im}\left(\lambda_{t}\right) \cdot F\left(x_{t}\right)
$$

\footnotetext{
${ }^{1}$ Later in another independent analysis [38], the global fit combining $B \rightarrow X_{s} \ell^{+} \ell^{-}, B \rightarrow K^{*} \mu^{+} \mu^{-}$, $B \rightarrow K \mu^{+} \mu^{-}$and $B_{s} \rightarrow \mu^{+} \mu^{-}$together obtained a similar constraint on $C_{10}$.

${ }^{2}$ The SM prediction is then $\mathcal{B}\left(K_{L} \rightarrow \mu^{+} \mu^{-}\right)_{\mathrm{SD}}=(0.79 \pm 0.12) \times 10^{-9}$, the experimental value in PDG is $\mathcal{B}\left(K_{L} \rightarrow \mu^{+} \mu^{-}\right)$exp. $=(6.84 \pm 0.11) \times 10^{-9}$.

${ }^{3}$ We should keep in mind that the original formula was obtained by comparing with two methods in SM [43]. A more serious formula in dark $Z$ model should be given by repeating this work similarly due to different types of $\Delta Y$, which is beyond the scope of current work. In the numerically study below, we will take $\Delta Y_{V}$ as an example. However, we will understand the exact form of $\Delta Y$ should keep the paper's conclusion.
} 
where $F(x)$ is given by

$$
F(x)=P_{0}+P_{X}\left[X_{0}(x)+\Delta X(x)\right]+P_{Y}\left[Y_{0}(x)+\Delta Y(x)\right]+P_{Z}\left[Z_{0}(x)+\Delta Z\right]+P_{E} E_{0}(x)
$$

and the factor $a=0.92 \pm 0.03$ [44], which takes into account the correction due to $\Delta I=5 / 2$ transitions [45]. Note the dark $Z$ modifies most parts of SM $F$ function but keep the gluon penguin vertex $E(x)$ unchanged. The coefficients $P_{i}(i=0, X, Y, Z, E)$ are given in terms of $R_{6}, R_{8}$

$$
P_{i}=r_{0}^{(0)}+r_{i}^{(6)} R_{6}+r_{i}^{(8)} R_{8}
$$

We adopt their numerical values for $\alpha_{s}\left(M_{Z}\right)=0.1185$ [46] given in table 1 of ref. [44]. For the nonperturbative parameters, we adopt the value $R_{8}=0.6, R_{6}=1.1$. The former one is obtained from lattice [47], with the translation by ref. [44]. But a reliable lattice result for $R_{6}$ is still lack, here we choose $10 \%$ deviation from large $\mathrm{N}$ result. The experimental value with $1 \sigma$ error for $\varepsilon^{\prime} / \varepsilon$ is

$$
\frac{\varepsilon^{\prime}}{\varepsilon} \simeq \operatorname{Re}\left(\frac{\varepsilon^{\prime}}{\varepsilon}\right)=(1.66 \pm 0.23) \times 10^{-3}
$$

taken form PDG [46].

\section{Results and discussion}

There are limited three free parameters in dark $Z$ model, $m_{Z^{\prime}}, \epsilon$ and $\epsilon_{Z}$. In our working scenario, the light $Z^{\prime}$ mass is ignored thus it might be promising to determine the remaining two by above observables. We take the global fit of Wolfenstein parametrization of CKM matrix as input since $Z^{\prime}$ does not change SM flavour structure. The other related input parameters have been given herebefore. Combining $B_{s} \rightarrow \mu^{+} \mu^{-}, K_{L} \rightarrow \mu^{+} \mu^{-}$and $\Delta C_{9}$, $\Delta C_{10}$ from a global fit of $B \rightarrow K^{(*)} \mu^{+} \mu^{-}$and so on, we plot allowed parameter space in $\left(\epsilon, \epsilon_{Z}\right)$ plane shown in figure 3.

The ranges of $\epsilon$ and $\epsilon_{Z}$ are both shown in the section $(-10,10)$. Due to its large uncertainty SD of $K_{L} \rightarrow \mu^{+} \mu^{-}$, as presented in grey region, gives a pretty wide band with $\frac{3}{5}$ slope. The green band with the same slope and less width of grey one, together with the gap out of this band surrounded by a set of hyperbolic curve, is resulted from $\Delta C_{10}$. Another parameter obtained from $B \rightarrow K^{(*)} \mu^{+} \mu^{-}$samely, $\Delta C_{9}$, constrains parameters in blue colour, which is restricted in two sets of hyperbolic curves and extended to slope $\frac{3}{5}$ direction as well as near vertical direction. Apparently the latter part is excluded by $K_{L} \rightarrow \mu^{+} \mu^{-}$(or $C_{10}$ ) while the former one is embedding in green area. Remarking in orange, the important $B_{s} \rightarrow \mu^{+} \mu^{-}$also embeds in $\Delta C_{10}$ but somehow has an overlap part with $\Delta C_{9}$. In principle, $B_{d} \rightarrow \mu^{+} \mu^{-}$can also be included. Considering its uncertainty is larger than $B_{s} \rightarrow \mu^{+} \mu^{-}$, the allowed region is then also wider than current orange area thus we do not show it. Here we also add in a typical constraint from $\varepsilon^{\prime} / \varepsilon$ by fixing its nonperturbative parameter $R_{6}$ and $R_{8}$. Showing as two narrow hyperbolic curves in yellow, it looks $\varepsilon^{\prime} / \varepsilon$ could give a very strict constraint. However, due to the large uncertainty of the two non-perturbative parameters, especially $R_{6}$, one cannot take the direct $\mathrm{CP}$ violation of 


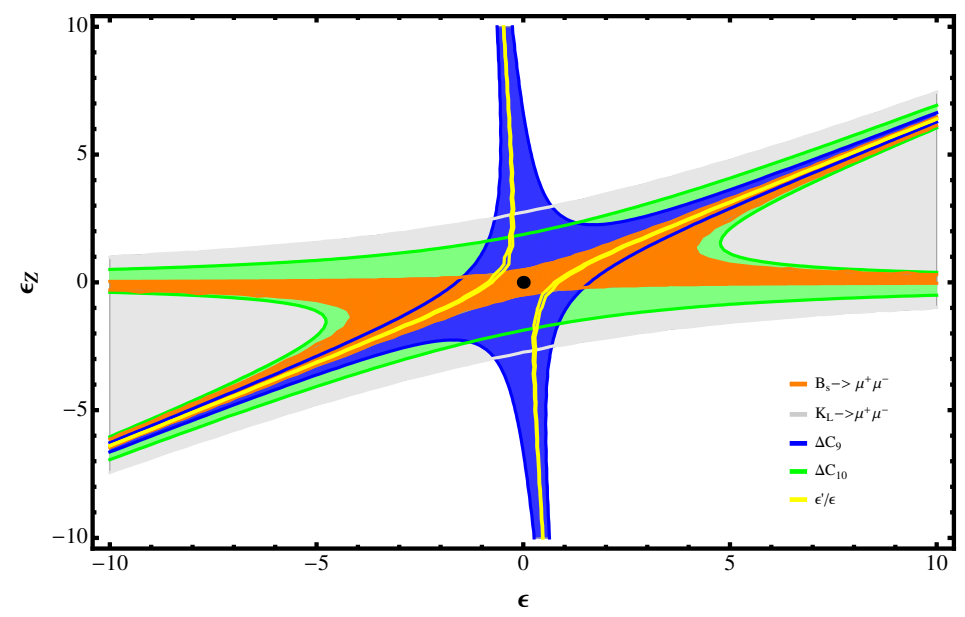

Figure 3. The allowed $\epsilon-\epsilon_{Z}$ parameter space by various experiments if $m_{Z^{\prime}}$ is less than $2 m_{\mu}$ and hence ignored. The meaning of colours are given as: grey stands for $K_{L} \rightarrow \mu^{+} \mu^{-}$, orange represents $B_{s} \rightarrow \mu^{+} \mu^{-}$, green denotes $\Delta C_{10}$, blue stands for $\Delta C_{9}$ while the yellow is one example of $\varepsilon^{\prime} / \varepsilon$ with $\left(R_{6}, R_{8}\right)=(0.7,1.1)$ and the black dot remarkes SM case.

kaon too seriously. Nevertheless, once $R_{6}$ is fixed within certain precisement in the future, $\varepsilon^{\prime} / \varepsilon$ can be taken as an important discrimination to further constrain the parameter.

Aiming at the determination of parameter space of dark $Z$ model, we reach the allowed $\left(\epsilon, \epsilon_{Z}\right)$ in the narrow linear region

$$
\epsilon_{Z}=\frac{3}{5} \epsilon
$$

and especially $-2<\epsilon<2,-1<\epsilon_{Z}<1$ are favored. In other words, the order of mixing parameters is constrained to be $\mathcal{O}(1)$. Generally speaking, more FCNC processes can be considered to determine the bound, but we can believe $\mathcal{O}(1)$ should be the typical value from flavour physics.

Many other works have already put constraints on dark photon model. Though it is not exact same model as our working scenario, the obtained mixing angle in dark photon still enlightens parameters in dark $Z$ model. For example, based on supernova 1987A the limit on mixing angle could be $\mathcal{O}\left(10^{-12.5}\right)$ for $m_{Z^{\prime}}>2 m_{e}$ [17]. A recent work from direct detection experiments such as XENON10 and XENON100, for the absence of an ionization signal, puts a even more stringent limit on $\epsilon$ down to $\mathcal{O}\left(10^{-15}\right)$. One may expect if $\epsilon_{Z}$ is added, the bound of $\epsilon$ will also be changed from astrophysical observables and direct detection experiements. Meanwhile, the working scenario in flavour physics can also be modified to allow $Z^{\prime}$ resonance production [15], leading to a possible different constraint on mixing parameters. In any case, given the bound from flavour physics in current scenario $\mathcal{O}(1)$, one may not expect a dramatical change (a more than 10 orders of magnitude) happens unnaturally.

In the existence of dark $Z$, we have investigated its effect in flavour physics especially by connecting the effect to the newly measured processes at LHC. However, the obtained bound, $\mathcal{O}(1)$, may not compete with the corresponding one in traditional dark matter study. 


\section{Acknowledgments}

FX would like to thank Dr. Xin-Qiang Li and Dr. Xiang-Dong Gao for the helpful discussion. This work is supported in part by the Ministry of Science and Technology of R.O.C. under Grant No. 104-2811-M-001-018 and National Natural Science Foundation of China under Grant No. 11405074.

\section{A The calculation of $Z^{\prime}$ penguin}

The effective $b s Z^{\prime}$ vertex is in the form of

$$
\Gamma_{\mu}\left(k_{1}, k_{2}\right)=e \lambda_{i} \frac{g_{2}^{2}}{2(4 \pi)^{2} m_{W}^{2}}\left[F_{1}\left(q_{\mu} \not-q^{2} \gamma_{\mu}\right) \mathbb{P}_{L}+F_{2} i \sigma_{\mu \nu} q^{\nu} m_{b} \mathbb{P}_{R}\right]
$$

which satisfies Ward Identity approximately in the limit of $m_{Z^{\prime}} \rightarrow 0$. In the phenomenology study of this work, we only focus on $F_{1}$ contribution, which has the relation with $H_{0}$ in section 3.1

$$
H_{0}(x)=-2 F_{1}(x)
$$

For convenience, in following calculation the effective vertex (A.1) can be rewritten as

$$
\Gamma^{\mu}\left(k_{1}, k_{2}\right)=e \lambda_{i} \frac{g_{2}^{2}}{2(4 \pi)^{2} m_{W}^{2}}\left[c_{L} T_{L}^{\mu}+c_{b} T_{b}^{\mu}+c_{s} T_{s}^{\mu}\right]
$$

by introducing $c_{b}=F_{1}+F_{2}, c_{s}=F_{2}-F_{1}, c_{L}=-F_{2}$ and $T_{L}^{\mu}=m_{b}^{2} \gamma^{\mu} \mathbb{P}_{L}, T_{b}^{\mu}=k_{1} k_{1}^{\mu} \mathbb{P}_{L}$, $T_{s}^{\mu}=\not k_{1} k_{2}^{\mu} \mathbb{P}_{L}$. Particularly we have

$$
F_{1}=\frac{1}{2}\left(c_{b}-c_{s}\right)
$$

In the calculation, the $F_{1}$ function may be projected to

$$
F_{1}=\left.\epsilon F_{1}\right|_{\gamma}+\left.\epsilon_{Z} F_{1}\right|_{Z}
$$

Following we will evaluate $F_{1}$ component by component.

\section{A.1 The $\epsilon$ component}

The contribution of $\epsilon$ component is exactly same as $b \rightarrow s \gamma$ case. Here we recalculate the photon penguin contribution. In Feynman-t' Hooft gauge, with the approximation $m_{s}=0$, $q^{2}=0$ and making use of quark on-shell condition as well as unitary CKM relation, we have the total contribution as

$$
\left.i \Gamma^{\mu}\right|_{\gamma}=\left.i e \lambda_{i} \frac{g_{2}^{2}}{2(4 \pi)^{2}} \sum_{j} \mathcal{M}_{j}^{\mu}\right|_{\gamma}
$$


with the individual amplitude from each diagram

$$
\begin{aligned}
&\left.\mathcal{M}_{a}\right|_{\gamma}=-\frac{4}{3}\left[\left(-2 C_{00}+m_{t}^{2} C_{0}-m_{b}^{2}\left(C_{0}+2 C_{1}+C_{2}+C_{11}+C_{12}\right)\right) T_{L}\right. \\
&\left.\quad+2\left(C_{11}+C_{1}\right) T_{b}+2\left(C_{0}+C_{1}+C_{2}+C_{12}\right) T_{s}\right] \\
&\left.\mathcal{M}_{b}\right|_{\gamma}=-\frac{2}{3} \frac{m_{t}^{2}}{m_{W}^{2}}\left[\left(-2 C_{00}+\frac{1}{2}+m_{t}^{2} C_{0}-m_{b}^{2}\left(C_{11}+C_{1}+C_{12}+C_{0}\right)\right) T_{L}\right.\left.\quad+2 C_{11} T_{b}+2\left(C_{12}-C_{2}\right) T_{s}\right] \\
&\left.\quad+\left(4 C_{11}+2 C_{1}\right) T_{b}+\left(4 C_{21}-2 C_{1}-2 C_{2}\right) T_{s}\right] \\
&\left.\mathcal{M}_{c}\right|_{\gamma}=- {\left[\left(12 C_{00}+m_{b}^{2}\left(3 C_{1}+2 C_{2}+2 C_{11}+2 C_{12}\right)\right) T_{L}\right.} \\
& \\
&\left.\mathcal{M}_{d}\right|_{\gamma}=-\frac{m_{t}^{2}}{m_{W}^{2}}\left[2 C_{00} T_{L}+\left(2 C_{11}+3 C_{1}+C_{0}\right) T_{b}+\left(2 C_{21}+C_{1}+2 C_{2}+C_{0}\right) T_{s}\right] \\
&\left.\mathcal{M}_{e}\right|_{\gamma}= m_{t}^{2} C_{0} T_{L} \\
&\left.\mathcal{M}_{f}\right|_{\gamma}=\left(m_{t}^{2} C_{0}+m_{b}^{2} C_{1}\right) T_{L}+2 C_{2} T_{s} \\
&\left.\mathcal{M}_{g}\right|_{\gamma}= \frac{1}{3}\left[\left(B_{0}+B_{1}\right)\left(2+\frac{m_{t}^{2}}{m_{W}^{2}}\right)-\frac{m_{t}^{2}}{m_{W}^{2}}\left(B_{0}\left(m_{b}^{2}, m_{W}^{2}, m_{t}^{2}\right)-B_{0}\left(0, m_{W}^{2}, m_{t}^{2}\right)\right)\right] T_{L}
\end{aligned}
$$

in which $B_{i}, C_{j}(i=0,1 ; j=0,1,2,11,12,21,22)$ are Pasarrino-Veltman integrals [48], and the positions for variables are assigned as $B\left(m_{b}^{2}, m_{W}^{2}, m_{t}^{2}\right), C_{a, b}\left(m_{b}^{2}, 0,0, m_{W}^{2}, m_{t}^{2}, m_{t}^{2}\right)$ for $a, b$ case, and $C_{c, d, e, f}\left(m_{b}^{2}, 0,0, m_{t}^{2}, m_{W}^{2}, m_{W}^{2}\right)$ for $c, d, e, f$ case. Note in the calculation, the light down type quark contribution in Goldstone-quark-quark vertex cannot be neglected.

The PV functions can be reduced into basic scalar function $B_{0}, C_{0}$, and we further perform Taylar expansion up to $m_{b}^{4}$. Sum up all the contribution together, we have

$$
\begin{aligned}
\left.i \Gamma^{\mu}\right|_{\gamma} & =i e \lambda_{i} \frac{g_{2}^{2}}{2(4 \pi)^{2} m_{W}^{2}}\left[\left(-\frac{1}{3}-2 \Delta_{\epsilon}\right) \frac{T_{L}}{m_{b}^{2}}+c_{L} T_{L}+c_{b} T_{b}+c_{s} T_{s}\right] \\
\left.c_{L}\right|_{\gamma} & =\frac{x_{t}^{2}\left(2-3 x_{t}\right) \ln x_{t}}{2\left(x_{t}-1\right)^{4}}-\frac{22 x_{t}^{3}-153 x_{t}^{2}+159 x_{t}-46}{36\left(x_{t}-1\right)^{3}} \\
\left.c_{b}\right|_{\gamma} & =\frac{\left(3 x_{t}^{4}-3 x_{t}^{3}+36 x_{t}^{2}-32 x_{t}+8\right) \ln x_{t}}{18\left(x_{t}-1\right)^{4}}+\frac{19 x_{t}^{3}-222 x_{t}^{2}+165 x_{t}-34}{108\left(x_{t}-1\right)^{3}} \\
\left.c_{s}\right|_{\gamma} & =\frac{\left(-3 x_{t}^{4}+57 x_{t}^{3}-72 x_{t}^{2}+32 x_{t}-8\right) \ln x_{t}}{18\left(x_{t}-1\right)^{4}}+\frac{113 x_{t}^{3}-696 x_{t}^{2}+789 x_{t}-242}{108\left(x_{t}-1\right)^{3}}
\end{aligned}
$$

The first term in eq. (A.8a) gives zero contribution after applying the unitary triangle relation $\sum_{i} \lambda_{i} \cdot$ constant $=0$. Also we have

$$
\begin{aligned}
\left.c_{b}\right|_{\gamma}-\left.c_{s}\right|_{\gamma} & =\frac{\left(3 x_{t}^{4}-30 x_{t}^{3}+54 x_{t}^{2}-32 x_{t}+8\right) \ln x_{t}}{9\left(x_{t}-1\right)^{4}}+\frac{-47 x_{t}^{3}+237 x_{t}^{2}-312 x_{t}+104}{54\left(x_{t}-1\right)^{3}} \\
& =-2\left(D_{0}\left(x_{t}\right)+\frac{26}{27}\right) \\
\left.F_{1}\right|_{\gamma} & =-D_{0}\left(x_{i}\right)
\end{aligned}
$$


in which $D_{0}\left(x_{i}\right)$ is vertex function of virtual photon in $b \rightarrow s \gamma$. Note in my calculation, the $D_{0}, D_{0}^{\prime}$ differs from the one in [28], up to a minus sign, for the different convention in QED vertex.

\section{A.2 The $\epsilon_{Z}$ component}

The total contribution for $b \rightarrow s Z$ is

$$
\left.i \Gamma^{\mu}\right|_{Z}=\left.i \frac{g_{2}^{2}}{2(4 \pi)^{2}} \frac{g_{2}}{c_{W}} \lambda_{i} \sum_{j} \Gamma_{j}^{\mu}\right|_{Z}
$$

Compared with $b \rightarrow s \gamma$ case, we only need to recalculate figure $(a)$ and figure $(b)$,

$$
\begin{aligned}
& \left.\Gamma_{a}^{\mu}\right|_{Z}=\frac{2}{3} s_{W}^{2} \cdot 2\left\{\left[-2 C_{00}+m_{t}^{2} C_{0}-m_{b}^{2}\left(C_{11}+C_{12}+2 C_{1}+C_{2}+C_{0}\right)\right] T_{L}^{\mu}\right. \\
& \left.+2\left(C_{11}+C_{1}\right) T_{b}^{\mu}+2\left(C_{12}+C_{2}+C_{1}+C_{0}\right) T_{s}^{\mu}\right\} \\
& -\frac{1}{2} \cdot 2\left\{\left[-2 C_{00}-m_{b}^{2}\left(C_{11}+C_{12}+2 C_{1}+C_{2}+C_{0}\right)\right] T_{L}^{\mu}\right. \\
& \left.+2\left(C_{11}+C_{1}\right) T_{b}^{\mu}+2\left(C_{12}+C_{2}+C_{1}+C_{0}\right) T_{s}^{\mu}\right\} \\
& \left.\Gamma_{b}^{\mu}\right|_{Z}=\frac{2}{3} s_{W}^{2} \cdot \frac{m_{t}^{2}}{m_{W}^{2}}\left\{\left[-2 C_{00}+\frac{1}{2}+m_{t}^{2} C_{0}-m_{b}^{2}\left(C_{11}+C_{12}+C_{1}+C_{0}\right)\right] T_{L}^{\mu}\right. \\
& \left.+2 C_{11} T_{b}^{\mu}+2\left(C_{12}-C_{2}\right) T_{s}^{\mu}\right\} \\
& -\frac{1}{2} \cdot \frac{m_{t}^{2}}{m_{W}^{2}}\left\{\left[m_{t}^{2} C_{0}-m_{b}^{2}\left(C_{0}+C_{1}\right)\right] T_{L}^{\mu}-2 C_{2} T_{s}^{\mu}\right\}
\end{aligned}
$$

while the other contributions are obtained by the following replacement

$$
\begin{aligned}
\text { Figure }(c): & e \rightarrow g_{2} c_{W} \\
\text { Figure }(d): & e \rightarrow g_{2} \frac{1-2 s_{W}^{2}}{2 c_{W}} \\
\text { Figure }(e),(f): & e \rightarrow-\frac{g_{2} s_{W}^{2}}{c_{W}} \\
\text { Figure }(g): & -Q_{b} \rightarrow-\left(-\frac{1}{2}+\frac{1}{3} s_{W}^{2}\right)
\end{aligned}
$$

with the same PV function convention as in $b \rightarrow s \gamma$ case.

In this work, since we only consider the $\left(q_{\mu} \phi-q^{2} \gamma_{\mu}\right)$ term, thus only the coefficients of $T_{b}^{\mu}$ and $T_{s}^{\mu}$ are of interest. Especially they are listed individually,

$$
\begin{aligned}
\left.m_{W}^{2} \Gamma_{a}^{\mu}\right|_{Z}= & {\left[\frac{-5 x_{t}^{2}+22 x_{t}-5}{18\left(x_{t}-1\right)^{3}}+\frac{\left(1-3 x_{t}\right) \ln x_{t}}{3\left(x_{t}-1\right)^{4}}+s_{W}^{2}\left(\frac{2\left(5 x_{t}^{2}-22 x_{t}+5\right)}{27\left(x_{t}-1\right)^{3}}+\frac{4\left(3 x_{t}-1\right) \ln x_{t}}{9\left(x_{t}-1\right)^{4}}\right)\right] T_{b}^{\mu} } \\
+ & {\left[\frac{20 x_{t}^{2}-7 x_{t}-7}{18\left(x_{t}-1\right)^{3}}-\frac{\left(6 x_{t}^{2}-6 x_{t}+1\right) \ln x_{t}}{3\left(x_{t}-1\right)^{4}}\right.} \\
& \left.+s_{W}^{2}\left(-\frac{2\left(20 x_{t}^{2}-7 x_{t}-7\right)}{27\left(x_{t}-1\right)^{3}}+\frac{4\left(6 x_{t}^{2}-6 x_{t}+1\right) \ln x_{t}}{9\left(x_{t}-1\right)^{4}}\right)\right] T_{s}^{\mu}
\end{aligned}
$$




$$
\begin{aligned}
& \left.m_{W}^{2} \Gamma_{b}^{\mu}\right|_{Z}=s_{W}^{2}\left(\frac{2\left(2 x_{t}^{2}-7 x_{t}+11\right)}{27\left(1-x_{t}\right)^{3}}+\frac{4 x_{t} \ln x_{t}}{9\left(x_{t}-1\right)^{4}}\right) T_{b}^{\mu} \\
& +\left[\frac{x_{t}\left(x_{t}-3\right)}{4\left(x_{t}-1\right)^{2}}+\frac{x_{t} \ln x_{t}}{2\left(x_{t}-1\right)^{3}}+s_{W}^{2}\left(\frac{x_{t}\left(11 x_{t}^{2}-43 x_{t}+38\right)}{27\left(x_{t}-1\right)^{3}}-\frac{2\left(3 x_{t}-4\right) \ln x_{t}}{9\left(x_{t}-1\right)^{4}}\right)\right] T_{s}^{\mu} \\
& \left.m_{W}^{2} \Gamma_{c}^{\mu}\right|_{Z}=\left[\frac{-17 x_{t}^{2}-8 x_{t}+1}{18\left(x_{t}-1\right)^{3}}+\frac{x_{t}^{2}\left(x_{t}+3\right) \ln x_{t}}{3\left(x_{t}-1\right)^{4}}+s_{W}^{2}\left(\frac{17 x_{t}^{2}+8 x_{t}-1}{18\left(x_{t}-1\right)^{3}}-\frac{x_{t}^{2}\left(x_{t}+3\right) \ln x_{t}}{3\left(x_{t}-1\right)^{4}}\right)\right] T_{b}^{\mu} \\
& +\left[\frac{-38 x_{t}^{2}+43 x_{t}-11}{9\left(x_{t}-1\right)^{3}}+\frac{2 x_{t}^{2}\left(4 x_{t}-3\right) \ln x_{t}}{3\left(x_{t}-1\right)^{4}}\right. \\
& \left.+s_{W}^{2}\left(-\frac{2 x_{t}^{2}\left(4 x_{t}-3\right) \ln x_{t}}{3\left(x_{t}-1\right)^{4}}+\frac{38 x_{t}^{3}-43 x_{t}+11}{9\left(x_{t}-1\right)^{3}}\right)\right] T_{s}^{\mu} \\
& \left.m_{W}^{2} \Gamma_{d}^{\mu}\right|_{Z}=\left[\frac{x_{t}\left(x_{t}^{2}-8 x_{t}-17\right)}{72\left(x_{t}-1\right)^{3}}+\frac{x_{t}^{2}\left(x_{t}^{2}-3 x_{t}+6\right) \ln x_{t}}{12\left(x_{t}-1\right)^{4}}\right. \\
& \left.+s_{W}^{2}\left(\frac{x_{t}\left(x_{t}^{2}-8 x_{t}-17\right)}{36\left(1-x_{t}\right)^{3}}-\frac{x_{t}^{2}\left(x_{t}^{2}-3 x_{t}+6\right) \ln x_{t}}{6\left(x_{t}-1\right)^{4}}\right)\right] T_{b}^{\mu} \\
& +\left[\frac{x_{t}\left(23 x_{t}^{2}-22 x_{t}-13\right)}{72\left(x_{t}-1\right)^{3}}-\frac{\left(x_{t}^{2}+3 x_{t}-6\right) \ln x_{t}}{12\left(x_{t}-1\right)^{4}}\right. \\
& \left.+s_{W}^{2}\left(\frac{x_{t}\left(-23 x_{t}^{2}+22 x_{t}+13\right)}{36\left(x_{t}-1\right)^{3}}+\frac{x_{t}^{2}\left(x_{t}^{2}+3 x_{t}-6\right) \ln x_{t}}{6\left(x_{t}-1\right)^{4}}\right)\right] T_{s}^{\mu}
\end{aligned}
$$

$\left.m_{W}^{2} \Gamma_{e}^{\mu}\right|_{Z}=0$

$\left.m_{W}^{2} \Gamma_{f}^{\mu}\right|_{Z}=s_{W}^{2}\left(-\frac{1-3 x_{t}}{2\left(x_{t}-1\right)^{2}}-\frac{x_{t}^{2} \ln x_{t}}{\left(x_{t}-1\right)^{3}}\right) T_{s}^{\mu}$

$\left.m_{W}^{2} \Gamma_{g}^{\mu}\right|_{Z}=0$

Sum up together, we have

$$
\begin{aligned}
\left.c_{b}\right|_{Z}= & {\left[\frac{-35 x_{t}^{3}+156 x_{t}^{2}-213 x_{t}+20}{216\left(x_{t}-1\right)^{3}}+\frac{\left(-3 x_{t}^{4}+9 x_{t}^{3}-18 x_{t}^{2}+28 x_{t}-4\right) \ln x_{t}}{36\left(x_{t}-1\right)^{4}}\right.} \\
& \left.+c_{W}^{2}\left(\frac{19 x_{t}^{3}-222 x_{t}^{2}+165 x_{t}-34}{108\left(x_{t}-1\right)^{3}}+\frac{\left(3 x_{t}^{4}-3 x_{t}^{3}+36 x_{t}^{2}-32 x_{t}+8\right) \ln x_{t}}{18\left(x_{t}-1\right)^{4}}\right)\right] \\
\left.c_{s}\right|_{Z}= & {\left[\frac{-103 x_{t}^{3}+438 x_{t}^{2}-507 x_{t}+136}{216\left(x_{t}-1\right)^{3}}+\frac{\left(3 x_{t}^{4}-27 x_{t}^{3}+36 x_{t}^{2}-10 x_{t}+4\right) \ln x_{t}}{36\left(x_{t}-1\right)^{4}}\right.} \\
& \left.+c_{W}^{2}\left(\frac{113 x_{t}^{3}-696 x_{t}^{2}+789 x_{t}-242}{108\left(x_{t}-1\right)^{3}}+\frac{\left(-3 x_{t}^{4}+57 x_{t}^{3}-72 x_{t}^{2}+32 x_{t}-8\right) \ln x_{t}}{18\left(x_{t}-1\right)^{4}}\right)\right]
\end{aligned}
$$

Now we obtain the $\tilde{D}_{0}$ function as

$$
\begin{aligned}
\left.c_{b}\right|_{Z}-\left.c_{s}\right|_{Z}=2\left[\frac{34 x_{t}^{3}-141 x_{t}^{2}+147 x_{t}-58}{216\left(x_{t}-1\right)^{3}}+\frac{\left(-3 x_{t}^{4}+18 x_{t}^{3}-27 x_{t}^{2}+19 x_{t}-4\right) \ln x_{t}}{36\left(x_{t}-1\right)^{4}}\right. & \\
& \left.+c_{W}^{2}\left(\frac{-47 x_{t}^{3}+237 x_{t}^{2}-312 x_{t}+104}{108\left(x_{t}-1\right)^{3}}+\frac{\left(3 x_{t}^{4}-30 x_{t}^{3}+54 x_{t}^{2}-32 x_{t}+8\right) \ln x_{t}}{18\left(x_{t}-1\right)^{4}}\right)\right] . \\
\tilde{D}_{0}\left(x_{i}\right) \equiv- & \left.F_{1}\right|_{Z}=\frac{1}{2 s_{W} c_{W}}\left(\left.c_{b}\right|_{Z}-\left.c_{s}\right|_{Z}\right)
\end{aligned}
$$

Take into account $D_{0}$ and $\tilde{D}_{0}$ together, the vertex function for $Z^{\prime}$ penguin $H_{0}$ is produced. 
Open Access. This article is distributed under the terms of the Creative Commons Attribution License (CC-BY 4.0), which permits any use, distribution and reproduction in any medium, provided the original author(s) and source are credited.

\section{References}

[1] Planck collaboration, P.A.R. Ade et al., Planck 2013 results. I. Overview of products and scientific results, Astron. Astrophys. 571 (2014) A1 [arXiv:1303.5062] [INSPIRE].

[2] B. Holdom, Two U(1)'s and $\epsilon$ charge shifts, Phys. Lett. B 166 (1986) 196 [InSPIRE].

[3] P. Fayet, Light spin 1/2 or spin 0 dark matter particles, Phys. Rev. D 70 (2004) 023514 [hep-ph/0403226] [INSPIRE].

[4] D.P. Finkbeiner and N. Weiner, Exciting dark matter and the INTEGRAL/SPI $511 \mathrm{keV}$ signal, Phys. Rev. D 76 (2007) 083519 [astro-ph/0702587] [INSPIRE].

[5] N. Arkani-Hamed, D.P. Finkbeiner, T.R. Slatyer and N. Weiner, A theory of dark matter, Phys. Rev. D 79 (2009) 015014 [arXiv: 0810.0713] [InSPIRE].

[6] S. Andreas, M.D. Goodsell and A. Ringwald, Dark matter and dark forces from a supersymmetric hidden sector, Phys. Rev. D 87 (2013) 025007 [arXiv:1109.2869] [INSPIRE].

[7] J.-W. Cui, H.-J. He, L.-C. Lu and F.-R. Yin, Spontaneous mirror parity violation, common origin of matter and dark matter and the LHC signatures, Phys. Rev. D 85 (2012) 096003 [arXiv: 1110.6893] [INSPIRE].

[8] D. Fargion, M. Khlopov and C.A. Stephan, Cold dark matter by heavy double charged leptons?, Class. Quant. Grav. 23 (2006) 7305 [astro-ph/0511789] [INSPIRE].

[9] M.Y. Khlopov and C.A. Stephan, Composite dark matter with invisible light from almost-commutative geometry, astro-ph/0603187 [INSPIRE].

[10] J.M. Cline, Z. Liu and W. Xue, Millicharged atomic dark matter, Phys. Rev. D 85 (2012) 101302 [arXiv:1201.4858] [InSPIRE].

[11] F.-Y. Cyr-Racine and K. Sigurdson, Cosmology of atomic dark matter, Phys. Rev. D 87 (2013) 103515 [arXiv:1209.5752] [INSPIRE].

[12] S. Andreas, M.D. Goodsell and A. Ringwald, Hidden photons in connection to dark matter, AIP Conf. Proc. 1563 (2013) 114 [arXiv: 1306.1168] [INSPIRE].

[13] K. Petraki, L. Pearce and A. Kusenko, Self-interacting asymmetric dark matter coupled to a light massive dark photon, JCAP 07 (2014) 039 [arXiv:1403.1077] [INSPIRE].

[14] K.-W. Ng, H. Tu and T.-C. Yuan, Dark photons as fractional cosmic neutrino masquerader, JCAP 09 (2014) 035 [arXiv: 1406.1993] [INSPIRE].

[15] H. Davoudiasl, H.-S. Lee and W.J. Marciano, 'Dark' Z implications for parity violation, rare meson decays and Higgs physics, Phys. Rev. D 85 (2012) 115019 [arXiv:1203.2947] [INSPIRE].

[16] PAMELA collaboration, O. Adriani et al., An anomalous positron abundance in cosmic rays with energies 1.5-100 GeV, Nature 458 (2009) 607 [arXiv:0810.4995] [INSPIRE].

[17] D. Kazanas, R.N. Mohapatra, S. Nussinov, V.L. Teplitz and Y. Zhang, Supernova bounds on the dark photon using its electromagnetic decay, Nucl. Phys. B 890 (2014) 17 [arXiv: 1410.0221] [INSPIRE]. 
[18] R. Foot and S. Vagnozzi, Dissipative hidden sector dark matter, Phys. Rev. D 91 (2015) 023512 [arXiv: 1409.7174] [InSPIRE].

[19] H. An, M. Pospelov, J. Pradler and A. Ritz, Direct detection constraints on dark photon dark matter, arXiv:1412.8378 [INSPIRE].

[20] BABAR collaboration, J.P. Lees et al., Search for a dark photon in $e^{+} e^{-}$collisions at BaBar, Phys. Rev. Lett. 113 (2014) 201801 [arXiv:1406.2980] [INSPIRE].

[21] O. Moreno, The heavy photon search experiment at Jefferson Lab, arXiv:1310.2060 [INSPIRE].

[22] PHENIX collaboration, A. Adare et al., Search for dark photons from neutral meson decays in $p+p$ and $d+A u$ collisions at $\sqrt{s_{N N}}=200 \mathrm{GeV}$, Phys. Rev. C 91 (2015) 031901 [arXiv: 1409.0851] [INSPIRE].

[23] W.-S. Hou, M. Kohda and F. Xu, Measuring the fourth generation $b \rightarrow s$ quadrangle at the LHC, Phys. Rev. D 84 (2011) 094027 [arXiv:1107.2343] [INSPIRE].

[24] LHCb collaboration, Differential branching fraction and angular analysis of the decay $B^{0} \rightarrow K^{* 0} \mu^{+} \mu^{-}$, JHEP 08 (2013) 131 [arXiv:1304.6325] [INSPIRE].

[25] S.D. Aristizabal, F. Staub and A. Vicente, Shedding light on the $b \rightarrow s$ anomalies with a dark sector, arXiv: 1503.06077 [INSPIRE].

[26] H. Ruegg and M. Ruiz-Altaba, The Stueckelberg field, Int. J. Mod. Phys. A 19 (2004) 3265 [hep-th/0304245] [INSPIRE].

[27] W.-Z. Feng, G. Shiu, P. Soler and F. Ye, Building a Stückelberg portal, JHEP 05 (2014) 065 [arXiv: 1401.5890] [INSPIRE].

[28] A.J. Buras, Weak Hamiltonian, CP-violation and rare decays, hep-ph/9806471 [INSPIRE].

[29] CMS and LHCb collaborations, Observation of the rare $B_{s}^{0} \rightarrow \mu^{+} \mu^{-}$decay from the combined analysis of CMS and LHCb data, Nature 522 (2015) 68 [arXiv:1411.4413] [INSPIRE].

[30] W.-S. Hou, M. Kohda and F. Xu, Implication of possible observation of enhanced $B_{d}^{0} \rightarrow \mu^{+} \mu^{-}$decay, Phys. Rev. D 87 (2013) 094005 [arXiv:1302.1471] [inSPIRE].

[31] K. De Bruyn et al., Branching ratio measurements of $B_{s}$ decays, Phys. Rev. D 86 (2012) 014027 [arXiv: 1204.1735] [INSPIRE].

[32] K. De Bruyn et al., Probing new physics via the $B_{s}^{0} \rightarrow \mu^{+} \mu^{-}$effective lifetime, Phys. Rev. Lett. 109 (2012) 041801 [arXiv: 1204.1737] [INSPIRE].

[33] A.J. Buras, R. Fleischer, J. Girrbach and R. Knegjens, Probing new physics with the $B_{s} \rightarrow \mu^{+} \mu^{-}$time-dependent rate, JHEP 07 (2013) 077 [arXiv:1303.3820] [INSPIRE].

[34] CMS and LHCb collaborations, Combination of results on the rare decays $B_{(s)}^{0} \rightarrow \mu^{+} \mu^{-}$from the CMS and LHCb experiments, CMS-PAS-BPH-13-007, LHCb-CONF-2013-012 (2014).

[35] Heavy Flavor Averaging Group collaboration, Y. Amhis et al., Averages of b-hadron, c-hadron and $\tau$-lepton properties as of early 2012, arXiv:1207.1158 [INSPIRE].

[36] A.J. Buras, F. De Fazio and J. Girrbach, 331 models facing new $b \rightarrow s \mu^{+} \mu^{-}$data, JHEP 02 (2014) 112 [arXiv:1311.6729] [INSPIRE].

[37] W. Altmannshofer, P. Paradisi and D.M. Straub, Model-independent constraints on new physics in $b \rightarrow s$ transitions, JHEP 04 (2012) 008 [arXiv:1111.1257] [INSPIRE]. 
[38] D.M. Straub, Constraints on new physics from rare (semi-)leptonic B decays, arXiv: 1305.5704 [INSPIRE].

[39] G. Isidori and R. Unterdorfer, On the short distance constraints from $K_{L, S} \rightarrow \mu^{+} \mu^{-}$, JHEP 01 (2004) 009 [hep-ph/0311084] [INSPIRE].

[40] A.J. Buras et al., Patterns of flavour violation in the presence of a fourth generation of quarks and leptons, JHEP 09 (2010) 106 [arXiv:1002.2126] [INSPIRE].

[41] M. Gorbahn and U. Haisch, Charm-quark contribution to $K_{L} \rightarrow \mu^{+} \mu^{-}$at next-to-next-to-leading order, Phys. Rev. Lett. 97 (2006) 122002 [hep-ph/0605203] [INSPIRE].

[42] A.J. Buras and J. Girrbach, Towards the identification of new physics through quark flavour violating processes, Rept. Prog. Phys. 77 (2014) 086201 [arXiv: 1306.3775] [InSPIRE].

[43] G. Buchalla, A.J. Buras and M.K. Harlander, Penguin box expansion: flavor changing neutral current processes and a heavy top quark, Nucl. Phys. B 349 (1991) 1 [INSPIRE].

[44] A.J. Buras, F. De Fazio and J. Girrbach, $\Delta I=1 / 2$ rule, $\varepsilon^{\prime} / \varepsilon$ and $K \rightarrow \pi \nu \bar{\nu}$ in $Z^{\prime}(Z)$ and $G^{\prime}$ models with FCNC quark couplings, Eur. Phys. J. C 74 (2014) 2950 [arXiv:1404.3824] [INSPIRE].

[45] V. Cirigliano, A. Pich, G. Ecker and H. Neufeld, Isospin violation in $\epsilon^{\prime}$, Phys. Rev. Lett. 91 (2003) 162001 [hep-ph/0307030] [INSPIRE].

[46] Particle Data Group collaboration, K.A. Olive et al., Review of particle physics, Chin. Phys. C 38 (2014) 090001 [InSPIRE].

[47] T. Blum et al., Lattice determination of the $K \rightarrow(\pi \pi)_{I=2}$ decay amplitude $A_{2}$, Phys. Rev. D 86 (2012) 074513 [arXiv:1206.5142] [InSPIRE].

[48] G. 't Hooft and M.J.G. Veltman, Scalar one loop integrals, Nucl. Phys. B 153 (1979) 365 [INSPIRE]. 